\title{
Câncer de mama: fatores de risco e detecção precoce
}

\author{
Breast cancer: risk factors and early detection
}

Cáncer de la mama: factores de riesgo y detección precoz

\author{
Pamella Araújo da Silva', Sueli da Silva Riul' \\ ' Universidade Federal do Triângulo Mineiro, Curso de Graduação em Enfermagem. Uberaba-MG, Brasil.
}

Submissão: 12-08-2010 Aprovação: 08-01-2012

\begin{abstract}
RESUMO
Objetivou-se identificar fatores de risco, segundo o INCA, para câncer de mama, analisar conhecimento e realização do AEM, ECM e mamografia, e verificar relação entre idade e escolaridade com conhecimento e realização desses exames. Dados coletados, durante dez meses, por entrevista com mulheres em quimioterapia contra câncer de mama, analisados por freqüência estatística simples. Observou-se concordância com a minoria dos fatores de risco. Todos os exames eram conhecidos e praticados pela maioria, exceto a mamografia realizada pela metade. As mais velhas praticavam principalmente AEM e mamografia, e as mais jovens ECM. Houve relação diretamente proporcional entre conhecimento desses exames e escolaridade. Os profissionais de saúde precisam orientar a população sobre câncer de mama, para permitir tratamentos mais resolutivos e menos mutiladores. Descritores: Enfermagem; Neoplasias da mama; Fatores de risco; Diagnóstico precoce.
\end{abstract}

\begin{abstract}
The objective was to identify risk factors, according to INCA, for breast cancer, analyzing knowledge and BSE practice, mammography and ECM, and verify the relationship between age and education with knowledge and conducting such examinations. Data were collected during ten months, through interviews with women in chemotherapy against breast cancer, and analyzed by simple statistics. There was agreement with the minority of risk factors. All examinations were known and practiced by most, except mammography performed in half. The older ones mainly practiced BSE and mammography, and the younger ECM. There was a directly proportional relationship between knowledge and education of these exams. Health professionals need to guide the public about breast cancer, to allow more resolute and less mutilating treatments.
\end{abstract}

Key words: Nursing; Breast neoplasm; Risk factors; Early diagnosis.

\section{RESUMEN}

Se objetivó identificar factores de riesgo, según el INCA, para cáncer de la mama, analizar conocimiento y realización del AEM, ECM y mamografía, y verificar relación entre edad y escolaridad con conocimiento y realización de eses exámenes. Los datos fueran colectados, durante diez meses, por entrevista con mujeres en quimioterapia contra cáncer de mama, y analizados por frecuencia estadística sencilla. Se observó concordancia con la minoría de los factores de riesgo. Todos los exámenes eran conocidos y practicados por la mayoría, excepto la mamografía realizada por la mitad de las mujeres. Las mas ancianas practicaban principalmente AEM y mamografía, y las más jóvenes ECM. Hubo relación directamente proporcional entre conocimiento de eses exámenes y escolaridad. Los profesionales de salud necesitan orientar la población sobre cáncer de mama, para permitir tratamientos más resolutivos y menos mutiladores.

Descriptores: Enfermería; Neoplasias de la mama; Factores de riesgo; Diagnóstico precoz. 


\section{INTRODUÇÃO}

O perfil da morbi-mortalidade brasileira vem sofrendo uma mudança intensa, passando de doenças infecto-parasitárias a crônico-degenerativas, como o câncer ${ }^{(1)}$, tendo como principais causas as mudanças nos hábitos de vida e no perfil epidemiológico da população(2).

O câncer de mama representa a principal causa de morte por câncer em mulheres brasileiras, e em nível mundial cede o lugar apenas para o câncer de pulmão ${ }^{(3)}$, representando um grande problema de saúde pública em todo o mundo(2).

O Brasil tem acompanhado as altas taxas de incidência e mortalidade de câncer de mama dos países desenvolvidos, porém as medidas necessárias à prevenção, ao diagnóstico e ao controle da doença não têm sofrido o mesmo crescimento $^{(4)}$. Foi estimado, para o ano de 2010, aproximadamente 49.240 novos casos de câncer de mama, com risco de 49 casos a cada 100 mil mulheres, e uma sobrevida mundial de $61 \%$ após cinco anos ${ }^{(5)}$.

O câncer de mama é raro antes dos 35 anos, crescendo rápida e progressivamente com a idade ${ }^{(6)}$, sendo descoberto, principalmente, entre 40 e 60 anos $^{(4)}$. Há referência de que a doença vem atingindo um maior número de mulheres jovens ${ }^{(7)}$.

É um dos tipos de câncer mais temidos pelas mulheres, devido à sua alta frequência e efeitos psicológicos ${ }^{(6)}$, tais como: alterações da sexualidade e da imagem corporal, medo de recidivas, ansiedade, dor e baixa autoestima ${ }^{(3)}$.

Os principais sinais e sintomas de câncer de mama são nódulo na mama e/ou axila, dor mamária e alterações da pele que recobre a mama, como abaulamentos ou retrações com aspecto semelhante à casca de laranja ${ }^{(6)}$. Os cânceres de mama localizam-se, principalmente, no quadrante superior externo, e em geral, as lesões são indolores, fixas e com bordas irregulares, acompanhadas de alterações da pele quando em estádio avançado ${ }^{(8)}$.

Os principais fatores de risco para o desenvolvimento de câncer de mama relacionam-se com idade avançada, características reprodutivas, história familiar e pessoal, hábitos de vida e influências ambientais. As características reprodutivas de risco se dão porque a doença é estrogênio-dependente ${ }^{(3)}$, e compreendem a menarca precoce (aos 11 anos ou menos), a menopausa tardia (aos 55 anos ou mais), a primeira gestação após os 30 anos e a nuliparidade ${ }^{(6)}$. A influência da amamentação, do uso de contraceptivos e da terapia de reposição hormonal (TRH) após a menopausa ainda são controversas ${ }^{(6)}$.

A história familiar e pessoal inclui as seguintes situações: um ou mais parentes de primeiro grau com câncer de mama antes dos 50 anos, um ou mais parentes de primeiro grau com câncer de mama bilateral ou câncer ovariano em qualquer idade, parente com câncer de mama masculina, câncer de mama e/ou doença mamária benigna prévios ${ }^{(4,6)}$. Os hábitos de vida relacionados são a obesidade, pelo aumento do nível de estrogênio produzido no tecido adiposo, principalmente no climatério ${ }^{(9)}$; uso regular de álcool acima de 60 gramas por dia, pois o acetaldeído, primeiro metabólito do álcool, é carcinogênico, mutagênico, estimulador da produção de estrogênio e imunodepressor ${ }^{(3)}$; e a influência do tabagismo ainda é controversa ${ }^{(8)}$. A principal influência ambiental é a exposição prévia às radiações ionizantes, sendo diretamente proporcional à dosagem da radiação e inversamente proporcional à idade da mulher na época da exposição(4).

Programas de prevenção primária evitam o aparecimento de doenças, mas não são utilizados em relação ao câncer de mama devido às suas características biológicas e recursos tecnológicos disponíveis ${ }^{(4)}$. O controle dessa doença se dá através da detecção precoce, na qual a lesão se restringe ao parênquima mamário, com um tamanho de no máximo três centímetros, permitindo o uso de recursos terapêuticos menos mutiladores e maior possibilidade de cura ${ }^{(10)}$. Os meios mais eficazes para a detecção precoce de câncer de mama são o exame clínico de mamas (ECM) e a mamografia ${ }^{(6)}$, pois o autoexame de mamas (AEM) detecta a doença geralmente em estádio avançado, sendo responsável por cerca de $80 \%$ das descobertas de cânceres de mama(4).

O AEM não tem efeito adverso e possibilita a participação da mulher no controle de sua saúde, devendo ser realizado mensalmente, entre o sétimo e o décimo dia após a menstruação, nos quais as mamas encontram-se indolores, menores e menos consistentes ${ }^{(4)}$. Nos casos de climatério, histerectomia e amamentação, quando não há menstruação, deve-se realizar o exame mensalmente, sempre no mesmo dia, escolhido arbitrariamente $^{(4)}$. As desvantagens do AEM são o maior número de biópsias de lesões benignas, falsa sensação de segurança nos exames falso-negativos e impacto psicológico nos falso-positivos ${ }^{(4)}$.

O ECM faz parte do atendimento integral à mulher, devendo ser inserido no exame físico e ginecológico de todas as mulheres, independente da faixa etária, servindo de subsídio para exames complementares ${ }^{(10)}$. A mamografia é um exame radiológico, de alto custo, dos tecidos moles das mamas, mais usado em mulheres com 35 anos ou mais, que permite a identificação de alterações não perceptíveis ao ECM, não substituindo o mesmo ${ }^{(4)}$. A ultrassonografia é o exame de escolha para mulheres com menos de 35 anos de idade, sendo também utilizado para mamas densas, nódulos palpáveis com/ sem mamografia negativa, processos inflamatórios e grávidas com sintomas mamários ${ }^{(4)}$.

O Instituto Nacional do Câncer (INCA) preconiza a realização do ECM anualmente, a partir dos 40 anos de idade; da mamografia, com intervalo máximo de dois anos, após os 50 anos; da combinação dos dois exames anualmente, a partir dos 35 anos, para os grupos com risco elevado; e a garantia de acesso ao diagnóstico, tratamento e seguimento para todas as mulheres com alteração nos exames realizados ${ }^{(10)}$.

As opções terapêuticas mais utilizadas para o tratamento locorregional do câncer de mama são a cirurgia e a radioterapia, enquanto que para o sistêmico são a quimioterapia, a hormonioterapia e a imunoterapia ${ }^{(10)}$.

Devido à grande ocorrência de pacientes com câncer de mama, em tratamento quimioterápico na Enfermaria de Ginecologia e Obstetrícia, do Hospital de Clínicas da Universidade Federal do Triângulo Mineiro (EGO/HC/UFTM), questionamos quais fatores de risco estariam relacionados a tal quadro, identificando o conhecimento e a realização do autoexame 
de mama, exame clínico de mamas e mamografia por estas mulheres, relacionando-os com a idade e a escolaridade, a fim de propor ações de orientação quanto aos riscos e detecção precoce do câncer de mama para proporcionar tratamentos menos mutiladores e melhor sobrevida.

O presente estudo teve como objetivos identificar os fatores de risco, segundo o INCA, para câncer de mama entre mulheres em tratamento quimioterápico na EGO/HC/UFTM, identificar o conhecimento e a realização do autoexame de mama, exame clínico de mamas e mamografia por este grupo de mulheres, e verificar a relação entre o conhecimento e a realização do autoexame de mama, exame clínico de mamas e mamografia com a idade e a escolaridade destas mulheres.

\section{METODOLOGIA}

Trata-se de estudo prospectivo, transversal, exploratório e descritivo. A população de estudo compreendeu pacientes atendidas na $\mathrm{EGO} / \mathrm{HC} / \mathrm{UFTM}$, para tratamento quimioterápico contra câncer de mama. A coleta de dados iniciou-se após aprovação pelo Comitê de Ética em Pesquisa da Instituição, Protocolo n 992/07, no período de outubro de 2007 a julho de 2008. Após a obtenção do Termo de Consentimento Livre e Esclarecido, os dados foram coletados por meio de entrevista com as mulheres, pelas próprias pesquisadoras. A coleta foi realizada durante o tratamento mencionado. $\mathrm{O}$ instrumento de coleta de dados foi constituído de informações relativas à: identificação (iniciais, registro hospitalar e escolaridade), fatores de risco (idade, antecedentes menstruais, obstétricos, pessoais e familiares, e hábitos de vida), conhecimento e utilização do AEM, ECM e mamografia.

A verificação da relação entre conhecimento e realização das estratégias mencionadas, idade e escolaridade foi desenvolvida mediante a comparação simples das informações com base em frequências estatísticas. Não foi possível a aplicação de testes estatísticos em função da pequena casuística. Foram entrevistadas todas as pacientes submetidas ao referido tratamento, no período do estudo, acima de 18 anos e que concordaram em participar. A compilação dos dados foi realizada no Microsoft Excel. As variáveis quantitativas foram submetidas às medidas descritivas: média, desvio padrão, moda, mediana e amplitude. Para as variáveis qualitativas foram obtidas distribuições de frequência. Os dados foram discutidos com a literatura específica da área.

\section{RESULTADOS E DISCUSSÃO}

A população do estudo compreendeu 18 pacientes, não havendo recusa à entrevista. Com relação ao perfil sócio-demográfico, a maioria, dez pacientes $(55,55 \%)$ era procedente de Uberaba/MG e 11 pacientes $(61,11 \%)$, possuíam pelo menos o ensino fundamental incompleto, sendo um perfil diferenciado em relação às pacientes usuárias do Sistema Único de Saúde (SUS), característico de portadoras de câncer de mama, ou seja, com nível socioeconômico mais elevado.

Em relação ao tratamento quimioterápico realizado, 15 dessas mulheres $(83,33 \%)$ seguiam o protocolo de tratamento de
Epirrubicina e Ciclofosfamida (EC), que consiste no protocolo de primeira escolha para o tratamento de câncer de mama. Durante o período de estudo, cinco pacientes (27,77\%) precisaram retornar ao tratamento seguindo outros protocolos, sendo eles o Paclitaxel e o CMF (Ciclofosfamida, Metotrexato e 5-Fluoruacil). Não houve óbito durante o estudo.

A grande maioria das pacientes, $14(77,77 \%)$ descobriu o câncer de mama através do AEM, demonstrando a importância de sua realização, não só para o conhecimento do próprio corpo, mas também para a detecção precoce de alterações, como o câncer.

A média de idade foi de 49,66 anos, variando de 30 a 66 (desvio padrão de 11,42 , de 38,24 a 61,08 ), sendo que a grande maioria, 15 pacientes (83,33\%), possuía 40 anos ou mais, assim como a maioria das mulheres matriculadas no Hospital do INCA III para tratamento de câncer de mama em 2001, corroborando a informação de idade avançada como um fator de risco para a doença ${ }^{(11)}$. Apesar do câncer de mama ser infrequente em mulheres jovens, ocorrendo antes dos 40 anos de idade em $6,5 \%$ dos $\operatorname{casos}^{(12)}$, no presente estudo foi encontrado em pacientes de 30, 32 e 38 anos, correspondendo a $16,66 \%$ dos casos.

A menarca ocorreu em média aos 12,5 anos, variando de dez a 17 (desvio padrão de 1,72, de 10,78 a 14,22), sendo que a menarca precoce ocorreu em apenas seis (33,33\%) dos casos, refutando a maior probabilidade de desenvolvimento de câncer de mama em mulheres que tiveram a menarca aos 11 anos ou menos.

Do total de pacientes, dez (55,55\%) ainda não tinham apresentado menopausa. Dentre as que já haviam apresentado, a média de idade de início foi de 51,12 anos, variando de 48 a 54 (desvio padrão de 2,03, de 49,09 a 53,15), não ocorrendo menopausa tardia em nenhum dos casos, também discordando da literatura. Como a média de idade das mulheres foi de 49,66 anos, dez (55,55\%) ainda não tinham 50 anos durante o estudo, não se podendo concluir com qual idade elas entrariam no climatério.

Todas as pacientes tiveram pelo menos uma gestação, sendo que a idade da primeira gestação foi em média aos 23,88 anos, variando de 16 a 34 anos (desvio padrão de 4,86, de $19,02$ a 28,74$)$, sendo que para dez pacientes $(55,55 \%$ ) com 23 anos ou mais. O número de filhos foi de 3,16 em média, variando de um a 12 filhos (desvio padrão de 2,72, de 0,44 a $5,88)$, sendo que dez pacientes $(55,55 \%)$ tinham no máximo dois filhos. A literatura aponta como fatores de risco a nuliparidade e gestação após os 30 anos de idade ${ }^{(4)}$.

A amamentação, considerada como fator protetor quando praticada no mínimo por seis meses, foi relatada por 12 das pacientes $(66,66 \%)$, com uma média de 10,5 meses, variando de seis a 48 (desvio padrão de 12,35, de -1,85 a 22,85), sendo que sete delas $(38,88 \%)$ amamentaram no máximo um ano, porém esse fator protetor ainda não está bem esclarecido na literatura.

O uso de contraceptivos orais esteve presente no relato de dez $(55,55 \%)$ das pacientes, variando de um mês a 16 anos de uso, sendo que sete pacientes (38,88\%) fizeram o uso por no máximo dez anos. A terapia de reposição hormonal foi 
utilizada por apenas uma paciente, pelo período de um ano e meio. Esses fatores de risco também ainda não foram muito bem estabelecidos, como mostra o estudo com mulheres do Rio Grande do Sul no qual não foi encontrada relação entre o uso de contraceptivos orais e o câncer de mama, evidenciando-se um maior risco no subgrupo de mulheres usuárias por mais de cinco anos e idade superior a 45 anos, mas sem significância estatística ${ }^{(13)}$. Conforme revisão bibliográfica realizada, o uso de TRH não está recomendado para mulheres sobreviventes ao câncer de mama e, para aquelas que nunca tiveram a doença o seu uso deve ser analisado como risco/beneficio, devendo ser utilizado em baixas doses e por curto período de tempo, sendo reconsiderada em intervalos regulares ${ }^{(14)}$.

Com relação à história familiar, 17 pacientes (94,44\%) não possuíam parente de primeiro grau com câncer de mama, sendo que a única resposta sim correspondia a uma irmã com câncer de mama antes dos 50 anos de idade. Nenhuma paciente possuía parente de primeiro grau com câncer de mama bilateral, câncer ovariano ou com câncer de mama masculina. A história familiar como fator de risco para o câncer de mama é o mais aceito para a comunidade científica, pois dados na literatura confirmam que o risco aumenta de duas a três vezes quando a mulher possui mãe ou irmã com câncer de mama, sendo que se for ambas o risco aumenta mais, principalmente se foi diagnosticado em idade precoce ${ }^{(4)}$.

Cinco pacientes $(27,77 \%)$ apresentaram lesões benignas prévias e uma teve câncer de mama prévio, sendo que conforme a literatura o risco aumenta em cinco vezes para recidiva de câncer de mama(4). As pacientes não souberam especificar as lesões benignas prévias, não podendo ser comparado com a literatura.

Sobre o hábito alimentar, dez pacientes (55,55\%) referiram manter dieta rica em gordura animal e rica em fibras - 12 pacientes $(66,66 \%)$. Cinco pacientes $(27,77 \%)$ possuíam índice de massa corporal normal; cinco apresentavam sobrepeso $(27,77 \%)$; quatro, obesidade em grau I $(22,22 \%)$; e quatro obesidade em grau II $(22,22 \%)$. Conforme a literatura, a gordura animal é fator de risco para desenvolvimento de câncer de mama, principalmente quando consumidos na infância e adolescência, enquanto que a dieta rica em fibras constitui-se um fator de proteção ${ }^{(4)}$. Em estudo realizado em João Pessoa/ $\mathrm{PB}$, observou-se que o consumo de carne vermelha e frituras pode aumentar o risco para o desenvolvimento de câncer de mama, enquanto que a ingestão de frutas, feijão e leite podem atuar como protetores ${ }^{(15)}$.

Em nenhum caso houve relato de exposição prévia e contínua a radiações ionizantes, um fator de risco apontado pelo INCA.

Metade das pacientes referiu hábito de tabagismo em algum momento de sua vida, com uma média de uso de 10,62 cigarros por dia, variando de dois a 20 (desvio padrão de 7,68, de 2,95 a 18,3), numa média de uso de 13,66 anos, variando de dois a 36 meses (desvio padrão de 10,10, de 3,56 a 23,76$)$, sendo que uma paciente não sabia informar a quantidade utilizada por dia. Apenas uma paciente referiu etilismo, com ingestão de dez copos de cerveja por dia durante dez anos. O tabaco é apontado como fator de risco para o desenvolvimento de câncer em geral, principalmente de pulmão e colo de útero, não sendo diretamente relacionado ao câncer de mama. O etilismo, para o grupo em estudo, não se mostrou como fator de risco predominante entre as mulheres, mas supõe-se que veio a contribuir como um fator a mais para desenvolvimento de câncer de mama na mulher em questão.

Ao serem abordadas sobre o conhecimento e a prática do AEM, todas as pacientes referiram já ter ouvido falar e a grande maioria, 16 pacientes $(88,88 \%)$ o praticava. Em outro estudo, com pacientes consultadas no Programa de Mastologia do Hospital de Clínicas da Universidade Federal de Goiás, 75\% conheciam o AEM e a maioria (51\%) o praticava ${ }^{(16)}$.

Dentre as 16 mulheres que realizavam o AEM, a maioria $(87,50 \%)$ iniciou essa prática após os 35 anos de idade, da mesma forma que em estudo realizado no Ambulatório de Especialidades da Faculdade de Medicina de Jundiaí e de Unidades Básicas de Saúde, em que a maioria das mulheres que não realizavam o autoexame de mama possuíam menos de 35 anos de idade ${ }^{(17)}$. A grande maioria, 12 pacientes $(75 \%)$, realizava-o em qualquer época do mês, e pelo menos uma vez por mês 14 pacientes $(87,50 \%)$, enquanto que dados do mesmo estudo referem que $39,82 \%$ das mulheres realizavam o AEM pelo menos uma vez por mês ${ }^{(17)}$. Em estudo realizado na cidade de Pelotas/RS, demonstrou-se que $80,4 \%$ das muIheres realizavam o AEM pelo menos uma vez por mês. Esse conflito de dados pode estar associado ao diferente nível socioeconômico existentes entre as cidades de Jundiaí e Pelotas, em que Pelotas apresenta um nível de escolaridade maior que Jundiaí $^{(7)}$. Em nosso estudo, observa-se que apesar de a maioria das mulheres não conhecerem a periodicidade correta do AEM, a maioria o praticava pelo menos uma vez por mês.

Onze pacientes $(68,75 \%)$ realizavam o AEM abrangendo toda a mama, porém apenas duas $(12,50 \%)$ abrangiam também as regiões axilar e supra clavicular, sendo essas as principais áreas de identificação de linfonodos metastáticos. Nove pacientes $(56,25 \%)$ observavam as mamas em repouso no espelho, porém apenas cinco $(31,25 \%)$ as observavam com a movimentação dos braços, fazendo com que a qualidade do AEM fosse perdida, pois não conseguiriam observar abaulamentos ou retrações ao movimento dos membros superiores. Nenhuma paciente realizava todas as etapas do AEM corretamente. No momento da entrevista foram feitas orientações em relação à prática correta do AEM e sua importância, pois cabe ao profissional de saúde realizar orientações sobre a promoção de saúde e a prevenção de doenças. As pacientes que não realizavam o AEM possuíam 30 e 49 anos. Dentre as que o realizavam, sete pacientes $(43,75 \%)$ faziam pelo menos três etapas corretas, sendo a maioria, nove pacientes (56,25\%) com 44 anos ou menos. Dentre as que realizavam a maior parte do AEM de forma errada, cinco pacientes (55,55\%) tinham 60 anos ou mais. Isso demonstra que, apesar das mulheres terem iniciado a prática de AEM com 35 anos ou mais, a maioria que o praticava corretamente estava nas faixas etárias menores.

A literatura aponta ainda para a necessidade de se responder às demandas da velhice, o que impõe para este segmento populacional uma assistência específica e integral ${ }^{(18)}$. O significado do autoexame de mamas para mulheres em tratamento 
contra câncer de mama mostrou-se ser sinônimo de prevenção e cuidado com a própria saúde ${ }^{(19)}$.

Uma das pacientes que não realizava o AEM não tinha o ensino fundamental completo e outra não era alfabetizada. Dentre as que praticavam pelo menos três etapas do AEM corretamente, quatro pacientes $(57,14 \%)$ tinham pelo menos o ensino fundamental completo e, dentre as que praticavam a maior parte errada, seis pacientes $(66,66 \%)$ tinham apenas o ensino fundamental incompleto. Esses dados sugerem que quanto maior o nível de escolaridade maior é a prática correta do AEM.

Em relação ao ECM, 15 pacientes $(83,33 \%)$ já tinham ouvido falar a respeito e $14(77,77 \%)$ o realizavam. Dentre as 14 pacientes que se submetiam ao ECM, oito $(57,14 \%)$ o realizavam antes dos 35 anos de idade e 11 o realizavam pelo menos uma vez por ano $(78,57 \%)$, sendo encontradas alterações anteriores em três $(21,42 \%)$ dos casos. Em estudo realizado com mulheres trabalhadoras de indústrias têxteis de Botucatu/ SP, $85 \%$ se submetia ao ECM pelo menos uma vez por ano ${ }^{(20)}$.

Todas as pacientes que nunca tinham ouvido falar no ECM e/ou não o praticavam possuíam 60 anos ou mais. Esse fato faz supor que as mulheres com idade avançada tenham vergonha em se submeter ao ECM realizado por um profissional de saúde.

Dentre as que não se submetiam ao ECM conforme a periodicidade preconizada pelo Ministério da Saúde, de uma vez por ano, as idades foram de 30, 38 e 65 anos. Todas as pacientes que nunca tinham ouvido falar no ECM e/ou não o praticavam possuíam escolaridade de, no máximo, ensino fundamental incompleto, demonstrando a influência da maior escolaridade na realização do ECM.

Em relação à mamografia, 17 das pacientes $(94,44 \%)$ já tinham ouvido falar e nove $(50 \%)$ já haviam realizado o exame, sendo que, das que não haviam realizado, seis $(66,66 \%)$ possuíam 40 anos ou mais e já deveriam ter realizado a mamografia pelo menos uma vez, conforme preconizado pelo Ministério da Saúde.

Dentre as nove pacientes que já haviam realizado a mamografia, oito $(88,88 \%)$ realizaram após os 35 anos de idade e pelo menos uma vez por ano $(66,66 \%)$. Em três $(33,33 \%)$ dos casos haviam sido encontradas alterações na mamografia.

A maioria, seis das pacientes $(66 \%)$, que não realizava mamografia tinha menos de 50 anos e a mesma porcentagem corresponde àquelas que não realizavam mamografia e tinham no máximo ensino fundamental incompleto, também demonstrando a influência da escolaridade nas práticas de saúde.

\section{CONCLUSÕES}

A partir das respostas obtidas e dos resultados do presente estudo, observamos que alguns fatores de risco e proteção foram confirmados e outros refutados, o que pode ter sido ocasionado pela pequena população do estudo.

As pacientes em tratamento quimioterápico para câncer de mama na EGO/HC/UFTM possuem um perfil diferenciado em relação à maioria dos pacientes usuários do SUS, pois são principalmente da cidade de Uberaba e possuem pelo menos ensino fundamental incompleto, característico de pacientes com câncer de mama, enquanto observa-se que pacientes com câncer de colo uterino são de um nível socioeconômico inferior.

Em relação aos fatores de risco e de proteção apontados pelo INCA, observou-se no grupo em estudo uma concordância da ocorrência com idade avançada, uso de contraceptivo oral, presença de lesões benignas prévias, obesidade e dieta rica em gordura. Outros fatores não estiveram presentes entre a maioria das mulheres, como menarca precoce, menopausa tardia, nuliparidade, primeira gestação após os 30 anos de idade, amamentação, dieta rica em fibra, uso de TRH, história familiar de neoplasia, tabagismo, etilismo e exposição a radiações ionizantes.

O AEM mostrou-se como de conhecimento de todas as mulheres e de prática entre a maioria, porém nenhuma delas realizava todas suas etapas corretamente, demonstrando que há uma necessidade de maior informação à população feminina, passo a passo, sobre a realização do mesmo, de forma clara e concisa. A maioria das mulheres iniciava sua prática com 35 anos ou mais, sugerindo que nessa fase da vida elas passam a se preocupar mais com o seu corpo e a terem mais medo do aparecimento de doenças crônicas, como o câncer. A prática correta do AEM esteve presente entre as mulheres mais jovens. Apesar das mulheres mais velhas praticarem mais o AEM, essas também o realizavam de forma errônea.

O ECM foi apontado como prática frequente entre a maioria das mulheres estudadas, nos intervalos recomendados, estando mais prevalente entre as mais jovens, provavelmente pela vergonha das mulheres com idade mais avançada em submeter-se a esse exame.

De acordo com o Ministério da Saúde, as mulheres com 40 anos ou mais devem realizar a mamografia de dois em dois anos, contudo a maioria das mulheres participantes deste estudo, nessa idade nunca o havia feito. Isso pode se dever ao elevado custo do exame e das extensas filas de agendamento para realizá-lo pelo SUS.

Houve uma relação entre conhecimento do AEM, ECM e mamografia com a escolaridade, de tal forma que quanto maior a escolaridade maior seu conhecimento e sua prática.

\section{CONSIDERAÇÕES FINAIS}

Durante experiência em estágio extracurricular de dois anos em sessões de quimioterapia contra câncer ginecológico na EGO/HC/UFTM, foi possível perceber a importância da atuação do enfermeiro direcionada a essas mulheres. A maioria traz consigo muitas crenças sobre a doença, o tratamento quimioterápico e cuidados a serem tomados. É importante respeitar a cultura das pacientes, e ao mesmo tempo fazer orientações adequadas sobre a patologia, o tratamento, os efeitos colaterais, alimentação e as condutas em emergências. É necessário estabelecer um contato harmônico com essas pacientes, a fim de tornar o processo de tratamento o mais humanizado possível, para que se sintam seguras e acolhidas.

A maioria dessas pacientes referiu ter descoberto o câncer de mama através do AEM, porém nenhuma o realizava de forma totalmente correta. Podemos supor que os meios de comunicação, principalmente a televisão estejam informando 
sobre a importância da realização do AEM, contudo não explicando todas as suas etapas. É preciso estar atento ao fato de que as propagandas são direcionadas a um público de um nível de escolaridade mais alta já que não são totalmente claras e objetivas.

Durante as entrevistas realizadas, mais de $1 / 4$ das mulheres relataram que já haviam descoberto alterações na mama seja por AEM, ECM ou mamografia, porém o profissional de saúde não valorizou tal queixa ou achado clínico. É necessário que o profissional de saúde esteja realmente envolvido no processo de saúde-doença do paciente, realizando um atendimento integral e levando em consideração as queixas da pessoa. Acreditamos que dessa forma, o atendimento se torne mais humano e resolutivo.

\section{REFERÊNCIAS}

1. Haddad N, Silva MB. Mortalidade por neoplasmas em mulheres em idade reprodutiva - 15 a 49 anos - no estado de São Paulo, Brasil, de 1991 a 1995. Rev Assoc Med Bras 2001; 47(3): 221-30.

2. Paulinelli RR, Freitas JR, Curado MP, Souza AA. A situação do câncer de mama em Goiás, no Brasil e no mundo: tendências atuais para a incidência e a mortalidade. Rev Bras Saude Mater Infant 2003; 3(1): 17-24.

3. Cantinelli FS, Camacho RS, Smaletz O, Gonsales BK, Braguittoni E, Rennó JR. A oncopsiquiatria no câncer de mama: considerações a respeito de questões do feminino. Rev Psiquiatr Clín 2006; 33(3): 124-33.

4. Ministério da Saúde (BR). Instituto Nacional do Câncer. Ações de enfermagem para o controle do câncer. $3^{\mathrm{a}} \mathrm{ed}$. Rio de Janeiro: INCA; 2008.

5. Ministério da Saúde (BR). Instituto Nacional do Câncer. Estimativas 2010: incidência de câncer no Brasil. Rio de Janeiro: INCA; 2009.

6. Ministério da Saúde (BR). Instituto Nacional do Câncer. Falando sobre câncer de mama. Rio de Janeiro: INCA; 2002.

7. Sclowitz ML, Menezes AMB, Gigante DP, Tessaro S. Condutas na prevenção secundária do câncer de mama e fatores associados. Rev Saúde Pública 2005; 39(3): 340-9.

8. Smeltzer SC, Bare BG. Brunner e Suddarth Tratado de enfermagem médico-cirúrgica. $10^{\mathrm{a}}$ ed. Vol 3. Rio de Janeiro: Guanabara Koogan; 2006

9. Cibeira GH, Guaragna RM. Lipídio: fator de risco e prevenção do câncer de mama. Rev Nutr 2006; 19(1): 65-75.

10. Ministério da Saúde (BR). Instituto Nacional do Câncer. Controle de câncer de mama: documento de consenso. Rio de Janeiro: INCA; 2004.

11. Skaba MMVF. Compreendendo a trajetória de mulheres em busca do diagnóstico e tratamento do câncer de mama: uma perspectiva sócio-antropológica [tese]. Rio de Janeiro (RJ): Pós-graduação em Saúde da Mulher, Fundação Oswaldo Cruz, 2003.

12. Clagnan WS, Andrade JM, Carrara HHA, Tezzi DG, Reis FJC, Marana HRC et al. Idade como fator independente de prognóstico no câncer de mama. Rev Bras Ginecol Obstet 2008; 30(2): 67-74.

13. Tessaro S, Beria JU, Tomasi E, Barros AJ. Contraceptivos orais e câncer de mama: estudo de casos e controles. Rev Saúde Pública 2001; 35(1): 32-8.

14. Junior WJA. TRH e câncer de mama. Revista Brasileira de Mastologia 2004; 14(2): 49-50.

15. Lima FEL, Latorre MRDO, Costa MJC, Fisberg RM Diet and cancer in Northeast Brazil: evaluation of eating habits and food group consumption in relation to breast cancer. Cad. Saúde Pública 2008; 24(4): 820-828.

16. Freitas JR, Koifman S, Santos NRM, Nunes MOA, Melo GG, Ribeiro ACG et al. Conhecimento e prática do autoexame de mama. Rev Assoc Med Bras 2006; 52(5): 337-41.

17. Borges JBR, Morais SS, Borges TG, Guarisi R, Maia EMC, Paganotti JC et al. Perfil das mulheres no Município de Jundiaí quanto ao hábito do autoexame de mamas. Revista Brasileira de Cancerologia 2008; 54(2): 113-122.

18. Carvalho CMRG, Brito CMS, Nery IS, Figueiredo MLF. Prevenção de câncer de mama em mulheres idosas: uma revisão. Rev Bras Enferm 2009; 62(4): 579-82.

19. Nascimento TG, Silva SR, Machado ARM. Autoexame de mama: significado para pacientes em tratamento quimioterápico. Rev Bras Enferm 2009; 62(4): 557-61.

20. Ferreira MLM, Oliveira C. Conhecimento e significado para funcionárias de indústrias têxteis sobre prevenção do câncer do colo uterino e detecção precoce do câncer de mama. Revista Brasileira de Cancerologia 2006; 52(1): 5-15. 